\title{
Real-world visual texture cloning upon prolonged gaze fixation
}

\author{
Aleksey V. Belikov \\ School of Biological and Medical Physics, \\ Moscow Institute of Physics and Technology \\ belikov.research@gmail.com
}

It is widely acknowledged that, in order to provide a rich unified experience to our internal conscious observer, the brain constructs a sophisticated model of the world on the basis of fragmented pieces of information incoming from sensory organs. It has been long known that perceptual filling-in mechanisms exist to extrapolate missing information from adjacent visual fields, especially in the periphery [1, 2]. Recently, a uniformity illusion has been reported for primitive features, such as color, shape and motion, in which the content of central vision spreads to the periphery upon prolonged gaze fixation $[3,4]$. Here I report that similar illusion exists for real-world textures, such as grass and foliage, observed directly in a real environment or on a photographic image. The texture around the point of gaze fixation is perceptually cloned to cover the areas of similar texture and color. This illusion may be triggered by the fading of textural information in the periphery due to the Troxler effect [5-7]. This is a preliminary report and requires validation in multiple subjects and detailed characterization.

Keywords: visual illusion, uniformity illusion, Troxler fading, perceptual filling-in, visual texture, peripheral vision, gaze fixation

To experience the illusion, it is required to fixate the gaze on a suitable texture, such as grass or foliage, for at least 30 seconds, while minimizing blinking (Fig 1A). After approximately 15 seconds, wobbling or morphing or fading of the image should occur, and at approximately 30 seconds the texture pattern around the point of fixation should spread (be cloned) to the whole area of similar texture and color (Fig 1B). At this stage, small movement of the gaze around the point of fixation (microsaccades) puts slightly different texture images on the fovea, which results in simultaneous changing of the cloned textures across the whole area (as in kaleidoscope). High-resolution image suitable for full screen viewing is available in Supplementary. 

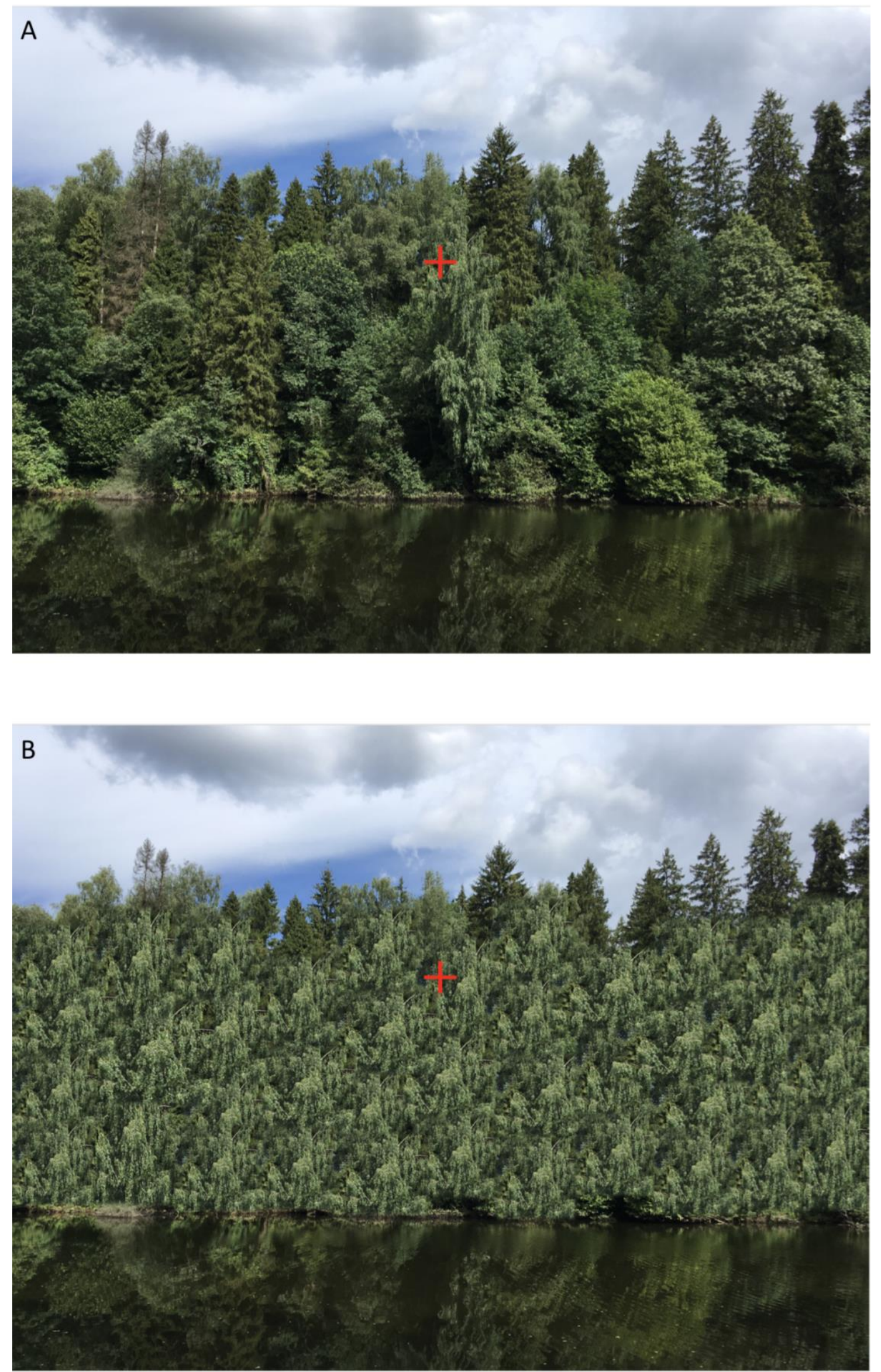

Figure 1. (A) The original scene on which gaze fixation was performed the position indicated by the red cross. (B) Approximate reconstruction of the observed illusion. 


\section{References}

1. Anstis S. Visual filling-in. Curr Biol 2010; 20: R664-666.

2. Weil RS, Rees G. A new taxonomy for perceptual filling-in. Brain Res Rev 2011; 67: 4055.

3. Otten M, Pinto Y, Paffen CL et al. The Uniformity Illusion. Psychol Sci 2017; 28: 56-68.

4. Uniformity Illusion. http://www.uniformillusion.com.

5. Martinez-Conde S, Macknik SL, Troncoso XG, Dyar TA. Microsaccades counteract visual fading during fixation. Neuron 2006; 49: 297-305.

6. Simons D, Lleras A, Martinez-Conde $S$ et al. Induced visual fading of complex images. J Vis 2006; 6: 1093-1101.

7. Troncoso XG, Macknik SL, Martinez-Conde S. Microsaccades counteract perceptual filling-in. J Vis 2008; 8: 15 11-19. 